\title{
Phase-Shift Induced in a High-Channel-Count Fiber Bragg Grating and Its Application to Multiwavelength Fiber Ring Laser
}

\author{
Xuxing Chen, Lunlun Xian, Kazuhiko Ogusu, and Hongpu Li
}

\begin{abstract}
A multi-channel filter formed by the piezoelectric transducer (PZT)-induced phase shift in a phase-only sampled FBG is experimentally demonstrated. The PZT-induced phase shift is stable and the magnitude of the induced phase shift is controllable. As an important application of this multichannel filter, a semiconductor optical amplifier (SOA) based multiwavelength fiber ring laser is successfully demonstrated.
\end{abstract}

Index Terms - Fiber Bragg gratings, phase shift, lasers.

\section{INTRODUCTION}

$\mathrm{T}$ He multi-channel filters with ultra-narrow bandwidth have attracted lots of interesting in the fields such as the wavelength selection, multiwavelength fiber laser and RF single generation. The ultra-narrow property of the filter can easily be realized by introducing a phase shift into a multi-channel fiber Bragg grating (FBG). To date, several kinds of Multi-channel FBG have been proposed and demonstrated, such as the sinc-sampled FBG, the superimposed FBG, the Talbot-effect based FBG and the amplitude-phase sampled FBG [1]. In particular, the phase-only sampled FBG, a specific case of the amplitude-phase sampled FBG, has the advantages of high channel counts, minimum index-modulation, high uniformity of inter-channel and compatibility with the standard of international telecommunications union (ITU) grids. On the other hand, a phase shift can be permanently inscribed into a FBG by using the phase shift phase mask technique [2], the UV post-processing [3], or the post-etching technique [4]. However, all of these approaches are lack of the flexibility for the implementation of both tunable and switchable devices. A temporary phase shift can be introduced into a FBG by the thermal method [5]-[7], where a narrow NiCr wire is utilized to heat the local region of a FBG. However, the thermal method

Manuscript received October 26, 2010; revised December 8, 2010. First published xxxxxx, 2011, current version published xxxxx, 2011. This work was supported in part by the Grant-in-Aid for Scientific Research from JSPS and in part by the International Communications Foundation in Japan.

$\mathrm{X}$. Chen is with the Graduate School of Science and Technology, Shizuoka University, L. Xian, K. Ogusu, and H. Li (e-mail: dhli@ipc.shizuoka.ac.jp) are with the Faculty of Engineering, Shizuoka University, Johoku 3-5-1, Hamamatsu 432-8561, Japan. needs high temperature to attain the $\pi$ phase shift. In addition, the temperature gradient and the expansion of the NiCr wire may make the phase shift not stable especially when the multiple wires are closely placed in order to generate multiple phase shifts in the FBG. Besides the thermal method, the mechanical stretch method can also be imposed to introduce a temporary phase shift in a FBG [8]-[11], which does not require complex temperature control. However, the strain- induced phase shift in a multi-channel FBG has not been reported.

In this study, the PZT-induced phase shift occurred in a linearly chirped 51-channel FBG is experimentally demonstrated. This induced phase shift is fairly stable and the magnitude of the induced phase shift can be controlled. As an important application of this multichannel filter, a semiconductor optical amplifier-based multi-wavelength fiber laser is successfully demonstrated. To the best of our knowledge, this is the first time to demonstrate a strain-induced phase shift on the multichannel FBG.

\section{PRINCIPLE AND EXPERIMENTAL SETUP}

Principle of the proposed approach is schematically shown in Fig. 1, where the PZT provided by the Nihon Ceramic with a size of $3 \times 3 \times 3 \mathrm{~mm}^{3}$ and the maximum extension-region of $1 \mu \mathrm{m}$ is wholly glued to the center of the phase-only sampled FBG along the grating direction. When the voltage is applied on the PZT, it will be extended. The accumulated phase change of the grating is then obtained at end point of the PZT and a narrow notch will be obtained in each channel of the grating due to the intrinsic properties of the sampled FBG. Unlike the thermal method [6], there is no influence of the temperature gradient and the thermal expansion. Therefore, multiple PZTs can be placed as close as possible to introduce multiple phase shifts in a FBG [9] as shown in Fig. 1. Moreover, tunability for the phase shift can easily be realized by adjusting the voltage applied on the PZT itself.

To confirm the proposal, we perform several tests for a phase-only sampled 51-channel FBG with one phase shift introduced by applying the voltage on a PZT. This grating is particularly designed with a dispersion magnitude of $1360 \mathrm{ps} / \mathrm{nm}$. The channel spacing is $0.8 \mathrm{~nm}$ exactly fitted with the ITU channel grids. The grating strength is about $10 \mathrm{~dB}$ with a length of about $12 \mathrm{~cm}$ and the flat-top bandwidth for each 
channel is about $0.6 \mathrm{~nm}$. Fig. 2 shows the reflection spectrum of five channels from $1551 \mathrm{~nm}$ to $1555 \mathrm{~nm}$, where the applied voltage imposing on the PZT is $150 \mathrm{~V}$. The generated notch in each channel has almost the same properties, i.e. the 3-dB bandwidth of the induced notch is about $0.028 \mathrm{~nm}$ and the notch depth is about $8 \mathrm{~dB}$. The inset in Fig. 2 shows an overall profile of 51-channel reflection spectrum. The stability of the PZT-induced multichannel notch filter is very important and we measure the reflection spectrum five times every other five minute. For clarity, Fig. 3 shows the five-channel reflection spectrum from $1551 \mathrm{~nm}$ to $1555 \mathrm{~nm}$ to reveal the stability of the resulted multichannel notch filter. No wavelength and reflection shift could be seen. The relation between the amount of the phase change and the notch depth is also investigated. Figure 4 shows the reflection spectrum calculated by the transfer matrix method when seven different phase-shifts ranged from 0 to $\pi$ are inserted at the center of the grating, respectively. The inset shows the details of the resulted spectrum. It can be seen that there really exists a stop-band (notch) at the center of each channel when phase shift is inserted. The notch-depth increases as the magnitude of the inserted phase is gradually increased and reaches the maximum only when $\pi$ phase shift is inserted. Meanwhile, the bandwidth of the notch decreases with increment of the phase shift. In our experiment, the amount of the PZT-induced phase shift can be easily controlled by changing the voltages imposed on the PZT as shown in Fig. 5. It can be seen that the magnitude related to the induced phase amount increases in accordance with the increment of the applied voltage, which agrees well with the simulation results. However, unlike the calculated ones, there also exists a side-notch appeared at the longer wavelength, which may be due to the birefringence of the grating and the asymmetric stretching of the PZT [9].

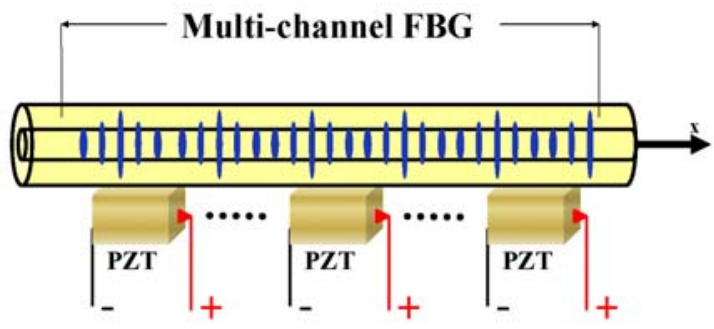

Fig. 1. Schematic diagram for the phase shifts induced by piezoelectric transducers (PZTs) in a multi-channel FBG.

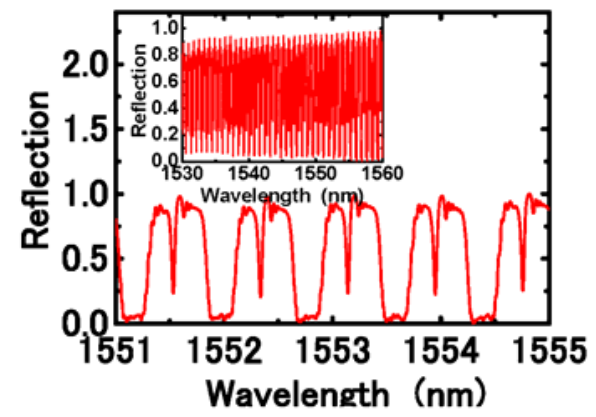

Fig. 2. Reflection spectrum for the particularly five channel notch filters. The inset shows overall profile of the 51-channel reflection spectrum.

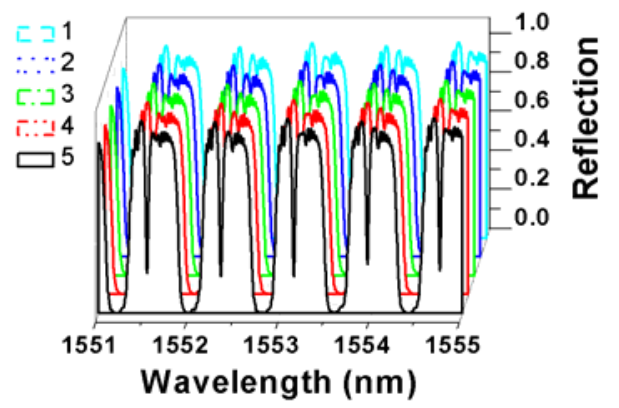

Fig. 3. Stability of the PZT-induced multichannel notch filter.

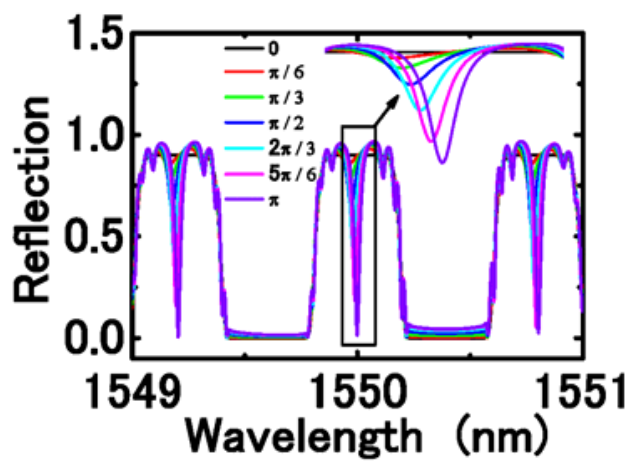

Fig. 4. The relation between the amount of the phase change and the notch depth calculated by the transfer matrix method.

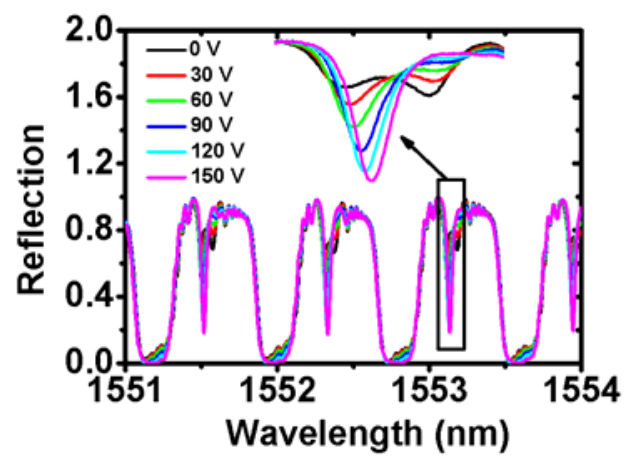

Fig. 5. The amount of the induced phase shift increases in accordance with the increment of the applied voltage.

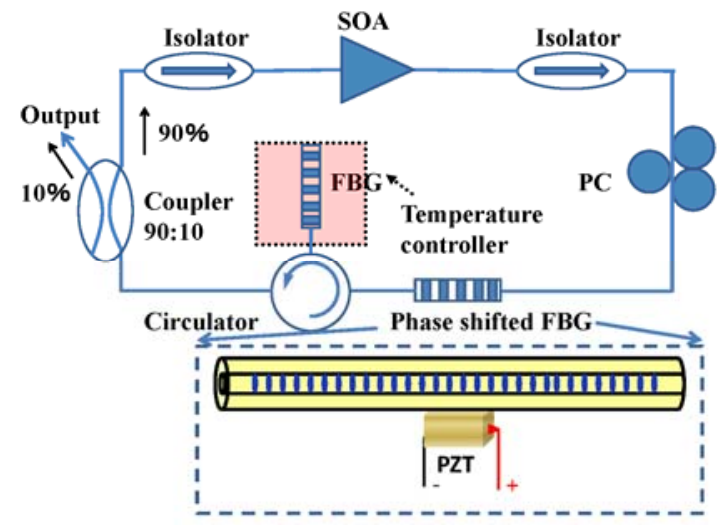

Fig. 6. Schematic diagram for the SOA-based multiwavelength fiber laser setup utilizing the PZT-induced phase shift. 
Next, by utilizing the phase shifted phase-only sampled FBG described above, a SOA-based multiwavelength fiber ring laser is demonstrated. Fig. 6 shows the schematic diagram of the experimental setup. Here the setup consists of a SOA (with a maximum small-signal gain of $19 \mathrm{~dB}$ and used as the gain medium), two isolators (to guarantee the unidirectional optical propagation), one polarization controller (PC), one 10/90 coupler, one circulator, and two concatenated phase-only sampled FBGs. Inset of the Fig. 6 shows the schematic diagram for the phase shift induced by a PZT. The multichannel band-pass filter with ultra-narrow bandwidth can be obtained by utilizing the two gratings which have identical characteristics but one of them (without the glued PZT) is placed in a temperature chamber and working in the reflection, and meanwhile the other one (with the phase-shift induced by PZT) is working in the transmission. Fig. 7 shows the measured 5-channel transmission spectrum for these two cascaded FBGs for clarity, where the voltage applied on the PZT is $150 \mathrm{~V}$. It can be seen that multichannel band-pass filter has been obtained and each of the channel has almost the same characteristics with a bandwidth of about $0.027 \mathrm{~nm}$ and an intensity height of about $7 \mathrm{~dB}$. The inset in Fig. 7 shows the entire spectrum of the all 51-channel band-pass filters. It can be seen that non-uniformity of the inter-channel transmission is less than $0.3 \mathrm{~dB}$. Figure 8 shows the measurement results for one typical lasing spectrum at room temperature. The inset shows the whole lasing spectrum. It can be seen that a multiwavelength $(>20)$ lasing with a channel spacing $0.8 \mathrm{~nm}$ has been obtained. Nonuniformity of the channel-to-channel output is about $20 \mathrm{~dB}$. The laser threshold for the SOA current is $250 \mathrm{~mA}$ and the output power is about $-20 \mathrm{dBm}$ at the SOA pump-current of $300 \mathrm{~mA}$. Each of the lasing has a $3-\mathrm{dB}$ linewidth of $0.016 \mathrm{~nm}$ and the signal-noise ratio is about $50 \mathrm{~dB}$. Stability for the output laser is also investigated. Spectrum for one typical lasing is scanned five times in every other five minute by using the optical spectrum analyzer (OSA) with a resolution of $0.01 \mathrm{~nm}$, which is shown in Fig. 8 also. No change for the lasing power and the peak wavelength can be found during the measurements, which in return means that a good stability for the proposed laser is realized.

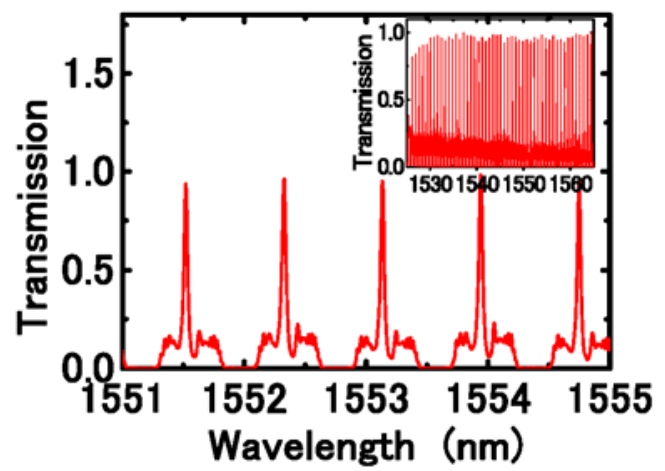

Fig. 7. Transmission spectrum for the two cascaded multi-channel FBGs. The inset shows the entire 51-channel transmission spectrum of the resulted band-pass filter.

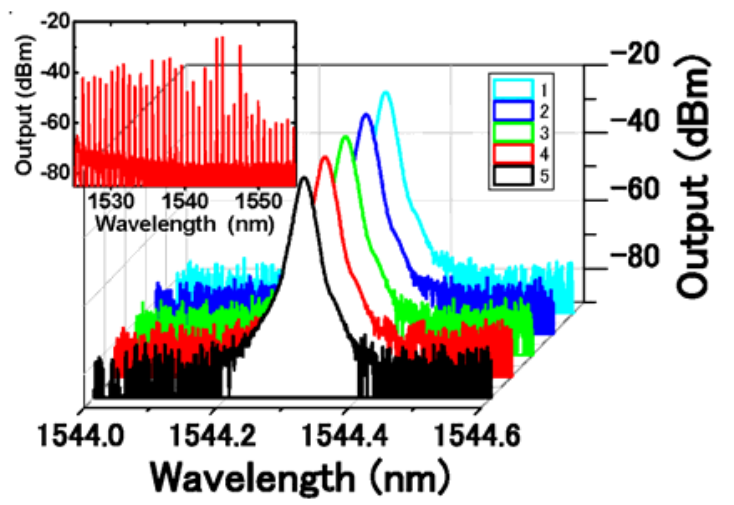

Fig. 8. Output spectra of the fiber ring laser measured in every five minutes.

\section{CONCLUSION}

In conclusion, a multi-channel filter formed by introducing a strain-induced phase shift into a phase only sampled FBG is firstly demonstrated. Based on this filter, a SOA-based multiwavelength fiber laser has been successfully demonstrated, which has potential application to fiber optic sensing for simultaneous measurement of multiple positions and multiple parameters [12].

\section{REFERENCES}

[1] H. Li, M. Li, Y. Sheng, and J. E. Rothenberg, "Advances in the design and fabrication of high-channel-count fiber Bragg gratings,” IEEE J. Lightw. Technol., vol. 25, pp. 2739-2750, 2007.

[2] R. Kashyap, P. Mckee, and D. Armes, "UV written reflection grating structures in photosensitive optical fibres using phase-shifted phase masks,” Electron. Lett., vol. 30, no. 23, pp. 1977-1978, 1994.

[3] J. Canning and M. Sceats, " $\pi$-phase-shifted periodic distributed structures in opticalfibres by UV post-processing,," Electron. Lett., vol. 30, no. 16, pp. 1344-1345, 1994.

[4] I. Del Villar, F. Arregui, I. Matias, A. Cusano, D. Paladino, and A. Cutolo, "Fringe generation with non-uniformly coated long-period fiber gratings,” Opt. Exp., vol. 15, no. 15, pp. 9326-9340, 2007.

[5] J. Kringlebotn, J. Archambault, L. Reekie, and D. Payne, " $\mathrm{Er}^{3+}: \mathrm{Yb}$ ${ }^{3+}$-codoped fiber distributed-feedback laser,” Opt. Lett., vol. 19, no. 24, pp. 2101-2103, 1994.

[6] S. Li, N. Ngo, S. Tjin, P. Shum, and J. Zhang, "Thermally tunable narrow-bandpass filter based on a linearly chirped fiber Bragg grating," Opt. Lett., vol. 29, no. 1, pp. 29-31, 2004.

[7] M. Li, H. Li, and Y. Painchaud, "Multi-channel notch filter based on a phase-shift phase-only sampled fiber Bragg grating,” Opt. Exp., vol. 16, no. 23, pp. 19388-19394, 2008

[8] M. Xu, A. Alavie, R. Maaskant, and M. Ohn, "Tunable fibre bandpass filter based on a linearly chirped fibreBragg grating for wavelength demultiplexing,” Electron. Lett., vol. 32, no. 20, pp. 1918-1919, 1996.

[9] X. Chen, Y. Painchaud, K. Ogusu and H. Li, "Phase shifts induced by the piezoelectric transducers attached to a linearly chirped fiber Bragg grating,” J. Lightw. Technol., vol. 28, no. 14, pp. 2017-2022, 2010.

[10] M. M. Ohn, A. T. Alavie, R. Maaskant, M. G. Xu, F. Bilodeau, and K. O. Hill, "Dispersion variable fiber Bragg grating using a piezoelectric stack," Electron. Lett., vol. 32, pp. 2000-2001, 1996.

[11] M. Pacheco, A. Mndez, F. M. Santoyo, and L. A. Zenteno, “Analysisof the spectral characteristics of piezoelectrically driven dual and triple period optical fiber Bragg gratings,” Opt. Commun., vol. 167, pp. 89-94, 1999.

[12] Q. Sun, D. Liu, L. Xia, J. Wang, H. Liu and P. Shum, "Experimental demonstration of multipoint temperature warning sensor using a multichannel matched fiber Bragg grating," IEEE Photon. Technol. Lett., vol. 20, no. 11, pp. 933-935, June. 2008. 